\title{
PREDICTIVE RESOURCE ALLOCATION FOR REAL TIME VIDEO TRAFFIC IN BROADBAND SATELLITE NETWORKS
}

\author{
H.O.Awadalla, L.G.Cuthbert and J.A.Schormans \\ Department of Electronic Engineering, Queen Mary \& Westfield College, Mile End Road, \\ London E14NS, UK. \{h.o.awadalla,l.g.cuthbert,j.a.schormans\}@elec.qmw.ac.uk
}

\begin{abstract}
Satellites are attractive for broadband networks because they provide fairly high bit-rate connections over a wide area. However, satellite networks are characterised by having a fixed amount of shared bandwidth between all users, and by relatively large propagation delays. These problems pose a challenge when implementing a high performance dynamic resource allocation scheme to effectively share the bandwidth among a large population of Earth terminals, with heterogeneous mix of traffic and different QOS requirements. This paper proposes the implementation of a predictive resource allocation scheme for the real time video component of the traffic in a broadband satellite network environment. Two predictive implementations are investigated: one based on time series analysis, and the other based on neural networks.
\end{abstract}

Keywords: ATM Networks, Broadband Satellite, Resource Allocation, Time Series Analysis, Neural Networks.

\section{INTRODUCTION}

Variable bit rate (VBR) video traffic is expected to be one of the major traffic types that need to be supported by broadband satellite networks. Broadband satellite networks will use ATM and most of the video encoding will be done using the MPEG standard (ISO Moving Picture Expert Group) [1]. Hung et al [2], have shown that for the up-link access, delay sensitive VBR connections should use both variable-rate and fixed-rate demand assignments. This is because variable-rate demand assignment on its own can cause excessive cell transfer delay (CTD) resulting in unacceptable cell losses at the receiving end. In Hung's scheme ( which we refer to as the classical scheme), the Earth terminals send the value of the occupancy of 
their buffers to the satellite. The occupancy of the terminal buffer can be defined by the following recursion [2]:

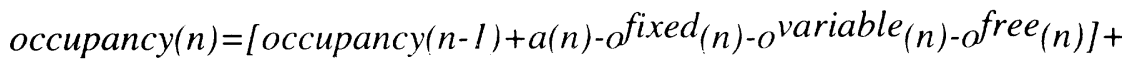

Where $a(n)$ is the number of cell arrivals to the terminal queue of a particular connection at the end of the nth TDMA frame, of ixed $_{(n)}$, $o^{\text {variable }_{(}(n) \text { and } o}$ free $_{(n)}$ are the number of slots assigned by fixed-rate DA, variable-rate $\mathrm{DA}$, and free assignment, respectively, to the connection on the uplink during the nth frame. Here, $x+=\max (x, 0)$. At time $(n+z)$, the satellite on-board scheduler makes the assignments for the up-link frame that will be received by the terminals at time $(n+2 z) . z$ is the propagation delay expressed in frames.

In this paper, we propose an implementation for a predictive resource allocation scheme where the assignment of the TDMA frame slots to the real-time VBR connections is performed according to the predicted MPEG frame values. One predictive implementation is based on time series analysis that is easy to incorporate, does not require any modification to the scheduler and does not require great computational complexity at the terminals. The other predictive implementation studied is based on neural networks and this requires greater computational complexity at the terminals, but like the time series analysis does not require modification to the scheduler.

Our results show that the time series analysis implementation is found to be comparable to the neural networks implementation in terms of the utilisation of both the ground and space segments resources.

\section{$1.1 \quad$ The Broadband Satellite Network}

This paper considers a broadband TDMA satellite communication system that uses an on-board packet switch in conjunction with an on-board scheduler to provide broadband services to a large population of Earth terminals. The Earth terminals can be both fixed and portable, with peak bit rates (up to $8 \mathrm{Mbit} / \mathrm{s}$ ) as shown in figure 1. TDMA is used for the up-link while TDM is used for the down-link. Residential and business applications that may use video encoding based on the MPEG standard include among other applications; direct-to-home video, telecommuting, distance learning and corporate training, collaborative group work and telemedicine. 


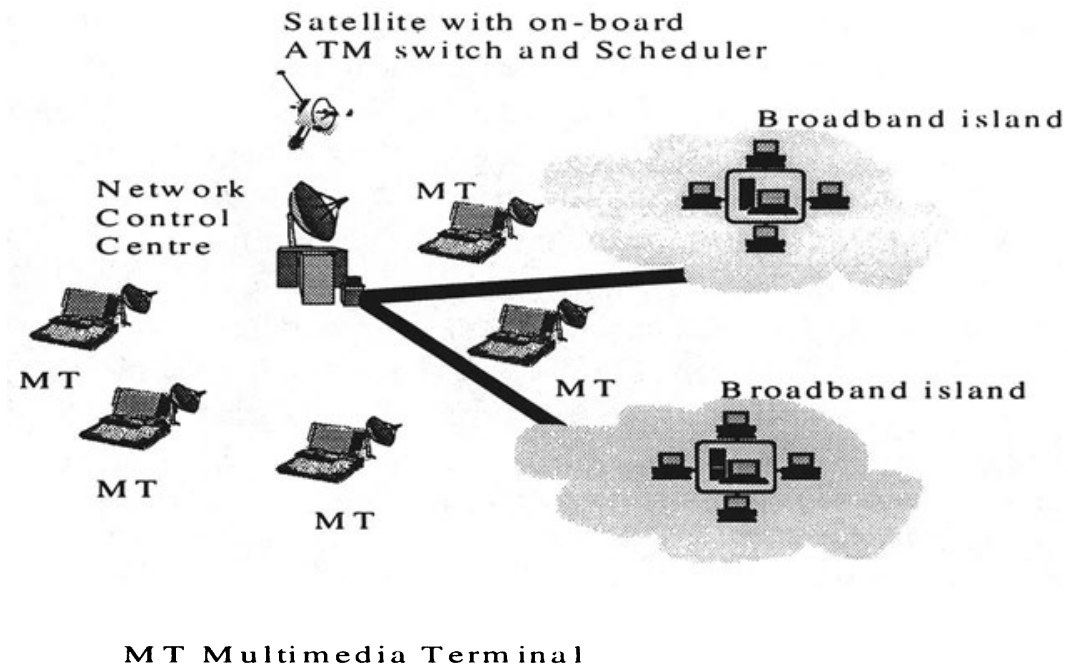

Figure 1 The broadband satellite network envisaged.

\section{PROPERTIES OF MPEG VIDEO DATA STREAMS}

The MPEG coding algorithm is now widely used in multimedia-video communications. An MPEG stream contains deterministic periodic sequences of frames referred to as a Group of Pictures (GOP) [3].

Frames can be encoded in three modes: intra-frames (I-frames), forward predicted frames (P-frames) and bi-directional predicted frames (B-frames) as shown in figure 2 .

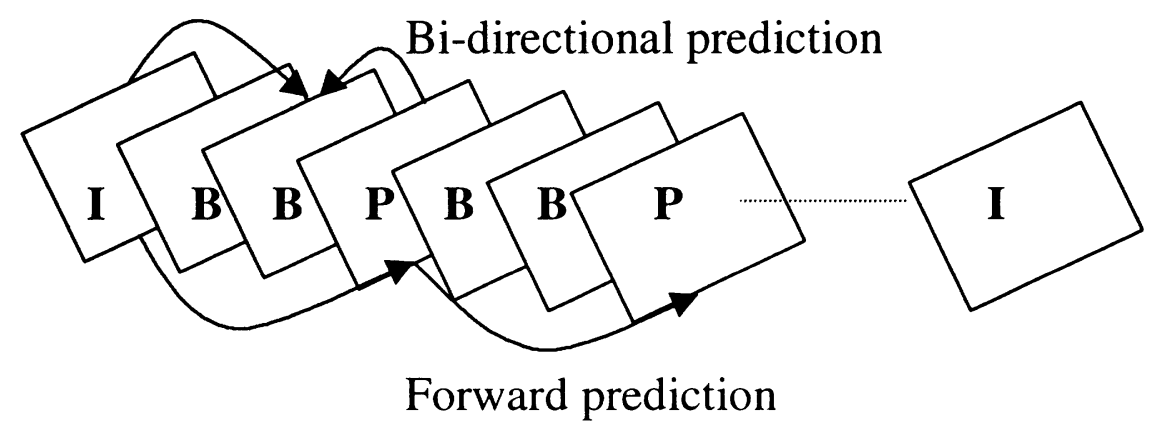

Figure 2 MPEG Group of Pictures (GOP) pattern 
An I-frame is encoded as a single image, with no reference to any past or future frames. The encoding scheme used is similar to JPEG compression for still images. A P-frame is encoded relative to the past reference frame, the future frame (which is the closest following I- or P-frame), or both. The encoding algorithm for B-frames is similar to P-frames, except that the motion vectors may refer to areas in the future reference frames.

In our work, we used two empirical MPEG-1 video traces from the movie Jurassic Park and a German "talk show". The traces vary in the degree of GOP-by-GOP correlation and frame-by-frame correlation as well as the scene activity. The traces are each about half an hour long and are available from the internet [4]. The sequence of MPEG I, P and B frames that was used is IBBPBBPBBPBB......(12 frames/GOP) and there are 40,000 frames in total per trace (each frame time is $40 \mathrm{~ms}$ ).

The statistical properties of the traces are shown below in Table 1:

\begin{tabular}{|l|c|c|}
\hline Sequence & Mean [Mbit/s] & Peak [Mbit/s] \\
\hline Jurassic Park & 0.33 & 1.01 \\
\hline Talk show & 0.36 & 1.00 \\
\hline
\end{tabular}

Table I Simple statistics of the encoded sequences

\section{IMPACT OF THE PREDICTIVE IMPLEMENTATION ON THE GROUND SEGMENT}

Implementing a predictive resource allocation scheme for the real time video connections at the ground segment (Earth terminals) entails modifications to the terminal design. Instead of generating the bandwidth demands of all connections at the terminal using Hung's recursion (equation 1), the demands of the real time video connections are generated using the predicted MPEG frames values. Hence the bandwidth generation process will have two flows as inputs: a continuous flow indicating the queue occupancy and another flow that is short lived (at connection set-up) from the real time video sources as shown in figure 3. This latter flow contains information about the MPEG properties of the video streams, e.g. the frames durations and GOP pattern. These properties are required by the predictive function which resides at the "Bandwidth requests generation" as shown in figure 3. 


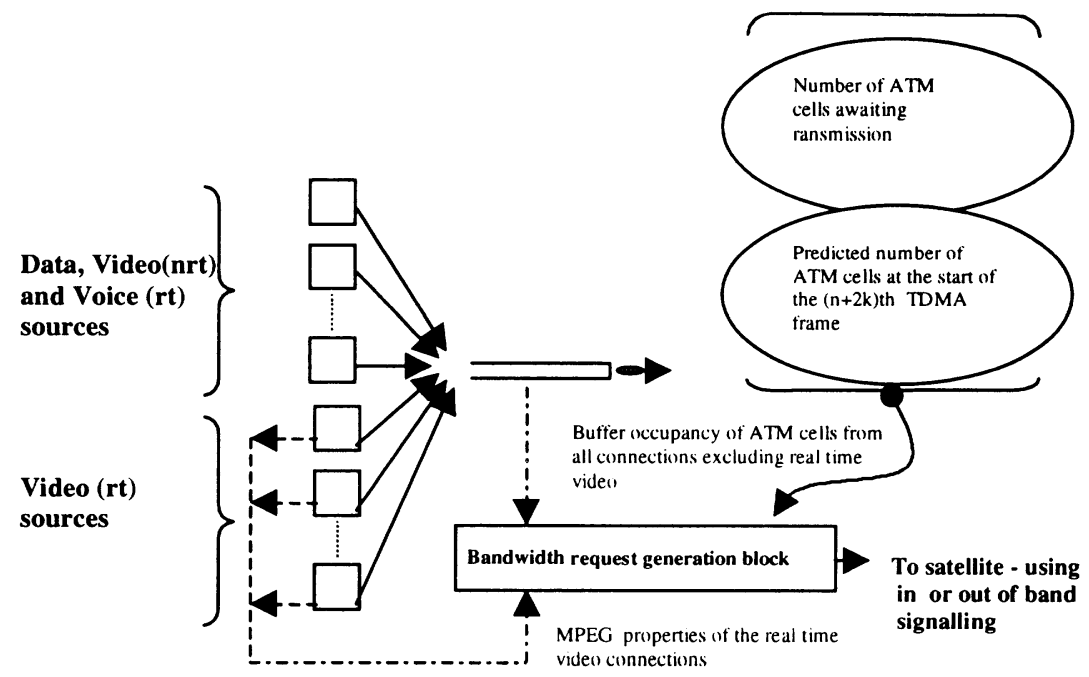

Figure 3 A conceptual design of the Earth terminal

\section{PREDICTION OF MPEG TRAFFIC USING TIME SERIES ANALYSIS}

In our proposed scheme, we assume that the MPEG stream is a time series $X(t)$ (that has seasonal effects as well as trends) generated by an additive model of the form:

$X(t)=T(t)+S(t)+R(t) \quad t=1,2, \ldots$

Here $T(t)$ is the trend, $S(t)$ the seasonal term, and $R(t)$ is the irregular or random term. We regard each frame of the GOP pattern as a unique seasonal term so that we have the following seasonal effects $S_{1}, S_{2}, \ldots . S_{12}$. Each seasonal effect, has period $s$; that is, it repeats after $s$ time periods:

$S(t+s)=S(t) \quad \forall t$

No trend was observed in the three traces, hence we set $T(t)$ to zero. We also set $R(t)$ to zero. The $j$ th seasonal is denoted as $S(j)$ and the period is taken as $s$. Then for the additive model we use an exponentially weighted moving average (EWMA) and, following [5], we have two smoothed series:

$M(t)=a \cdot(X(t-S(t-s))+(I-a) \cdot M(t-I)|a|<1$ 
$S(t)=b \cdot(X(t)-M(t))+(I-b) \cdot S(t-s) \quad|\mathrm{b}|<1$

Where $a$ and $b$ are the discounting parameters. The forecast of $X(t+k)$ made at time $t$ is:

$F(t+k)=M(t)+S(t+k-s)$

\section{PREDICTION OF MPEG TRAFFIC USING NEURAL NETWORKS}

The earth terminal must be able to predict at least $250 \mathrm{~ms}$ ahead to account for the round trip delay. A feed-forward three layered (30-15-10) Neural Network was trained on each trace. Fifteen successive MPEG frames were used as inputs to the Neural Network to predict the twenty third successive frame which starts after $(40 * 7)=280 \mathrm{~ms}$ from the end of the fifteenth frame, as shown in figure 4 . The extra $30 \mathrm{~ms}$ ensures that processing delays are catered for.

As there are no rules for choosing the number of inputs as well as the number of hidden layers and neurons, a number of experiments were required to achieve a near optimum configuration for the neural network. It was found that the more inputs the neural network has, the better the

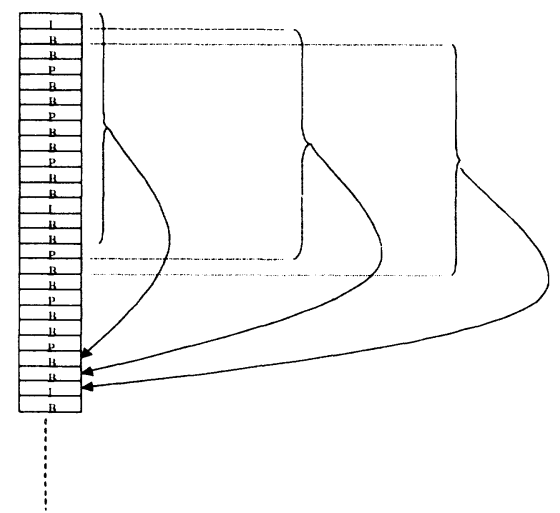

Figure 4 Prediction using Neural Networks 
prediction. However, it was also noticed that the improvement is not substantial for more than ten inputs. Also the more inputs and hidden layers, the greater the complexity that makes the implementation of the neural network at the earth terminal unattractive.

The learning algorithm used by the neural network was the Back Propagation [6] algorithm and the activation function was the sigmoid function, which is a commonly used activation function for back propagation neural networks. A training file of 5000 samples (1/9 of the whole MPEG trace) was used to train the neural network (this was carried out for each trace). All inputs were scaled so that they lay in the range (0-1). This was achieved by dividing all samples of each training file by the size of the largest frame in the respective trace.

\section{SIMULATION STUDIES AND RESULTS}

In the simulations, we assume that each MPEG trace starts at the same time as the start of a TDMA frame and that the MPEG video traffic is deterministically smoothed over the MPEG frame period, i.e. cells emitted during a frame period are evenly spaced. We can then write the forecast value of the integer number of cells that will arrive during the frame starting at ( $t+k+t d m a$ frame duration) as a result of the predicted MPEG frame as:

$E=\lceil F(t+k) . t$ dma frame duration/mpeg frame duration $\rceil$

$k$ in the case of the time series analysis is equal to $440 \mathrm{~ms}(11 * 40)$ and for the neural network case, it is equal to $280 \mathrm{~ms}\left(7^{*} 40\right)$.

To allow for under-prediction, we allocate $\phi$ number of fixed slots in every TDMA frame (or multiple of frames) to the real time video connection. The earth terminal signals a demand of:

$D=E+\phi$

for bandwidth to the satellite through inband or out-of-band signalling as opposed to signalling the buffer occupancy as is the case in the classical scheme. For simplicity we set the TDMA frame duration equal to the MPEG frame duration and size the TDMA frame slot to hold one ATM cell. 
The values of the different parameters in the simulation are all shown below in table 2 :

\begin{tabular}{|l|l|}
\hline Uplink capacity & $8 \mathrm{Mbit} / \mathrm{s}$ \\
\hline TDMA frame duration & $40 \mathrm{~ms}$ \\
\hline Round trip propagation delay (RTD) & $250 \mathrm{~ms}$ \\
\hline Discount parameter "a" & 0.075 \\
\hline Discount parameter "b" & 0.75 \\
\hline
\end{tabular}

Table 2 Simulation parameters values

When using time series analysis, large values of " $b$ " and small values of "a" make the smooth series react rather quickly to changes, hence the choice of "a" and "b". Also, in the time series analysis, when estimating the seasonal effects $\left(S_{1}, S_{2}, \ldots S_{12}\right)$, we consider the first two GOP pattern data and following [5] we take the seasonal minus the overall mean.

\subsection{Performance Objective}

The ground segment and space segment resources are the Earth terminal's buffer and the TDMA frame slots respectively.

To compare the proposed predictive and the classical schemes, a number of simulations were performed with different values of $o^{\text {fixed }}$ for the classical scheme and different values of $\phi$ for the predictive schemes. The performance objective in all three schemes was the same: a cell transfer delay of less than (RTD+TDMA frame duration), i.e. a cell delayed by more than one frame duration, at the sending terminal's buffer is discarded at the receiving terminal.

We define the relative mean allocation rate as the ratio of the mean allocation rate to the mean cell rate. Figures 5,6 and figures 7,8 show the cell discard probability and the mean buffer size when each of the three traces is considered (plotted against the relative mean allocation rate). It is apparent that the predictive schemes proposed here, requires less TDMA frame slots to achieve a specific performance objective as well as less buffer space than the classical scheme.

The time series analysis predictive implementation is less complicated than the neural network one and compares well with it, bearing in mind that 
the neural network predicts frames $280 \mathrm{~ms}$ ahead while the time series analysis scheme predicts frames $440 \mathrm{~ms}$ ahead.

A Neural Network has to be trained on a portion of the traffic to be able to predict accurately. If however, the traffic characteristic changes, the neural network may not be able to achieve reasonable performance.

For all the above reasons, we suggest that it will be more economical and feasible to implement the predictive scheme using time series analysis.

\section{CONCLUSIONS}

Two predictive implementations for resource allocation for real time video traffic in a satellite network, were presented. The implementations are based on time series analysis and Neural Networks.

Both implementations outperform the classical assignment scheme in the usage of both the ground and space segment resources of the satellite network. Moreover, they do not require extra computational effort from the satellite, which is vital if the scheduler is to be housed on board the satellite. However, the time series analysis implementation is more attractive than the Neural one as it needs minimum computational support at the Earth terminals. A conceptual design for the Earth terminal to cope with a predictive implementation for resource allocation has also been outlined.

\section{References}

[1] D.LE GALL: 'MPEG: A video compression standard for multimedia applications'. Communications of ACM, 34(4), April 1991, pp. 46-58

[2] A..HUNG, M.J.MONTPETITT, G.KESIDIS: ' A Framework for ATM via Satellite', IEEE GLOBECOM, London, Nov.1996, vol.2., pp.10201025

[3] O.ROSE: 'Statistical properties of MPEG video traffic and their impact on traffic modelling in ATM systems'. Research Report Series, Report No.101, February 1995, Institute of Computer Science,University of Wuerzburg, Germany.

[4] ftp-info3.informatik.uni-wuerzburg.de Directory: /pub/MPEG/traces

[5] G.JANACEK, L.SWIFT: 'Time Series: Forecasting,simulation,applications", Ellis Horwood series in mathematics and its application, Chichester, pp.14-32 
[6] D.E.RUMELHART, G.E.HINTON, R.J.WILLIAMS: 'Learning representations By Back-Propagating Error.' Nature, 1986, 323:533536. Reprinted in Anderson \& Rosenfeld, 1988, pp. 696-699

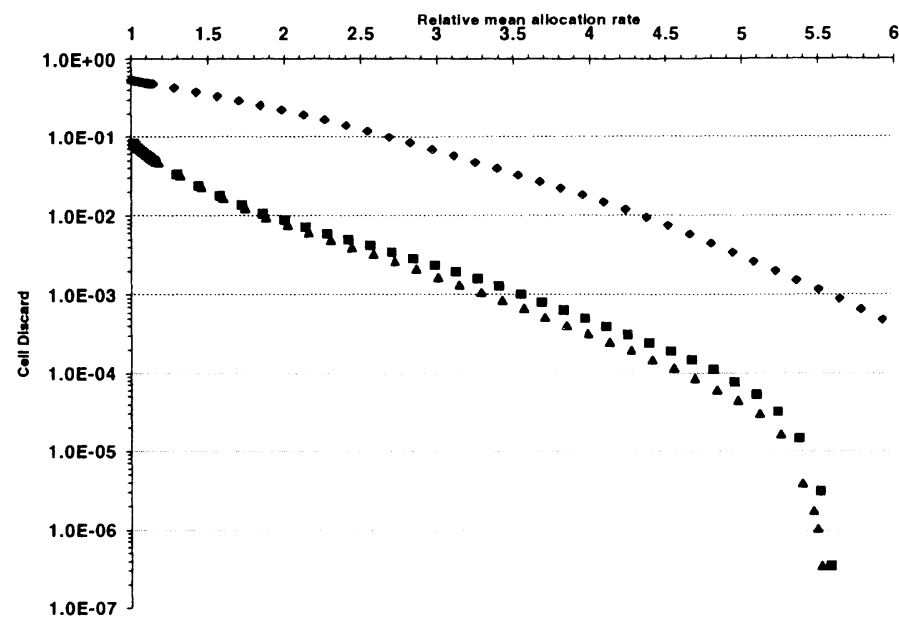

Classical scheme - Predictive "Time Series Analysis - EWMA" scheme

^ Predictive "Neural" scheme

Figure 5 Cell discard (Jurassic Park trace)

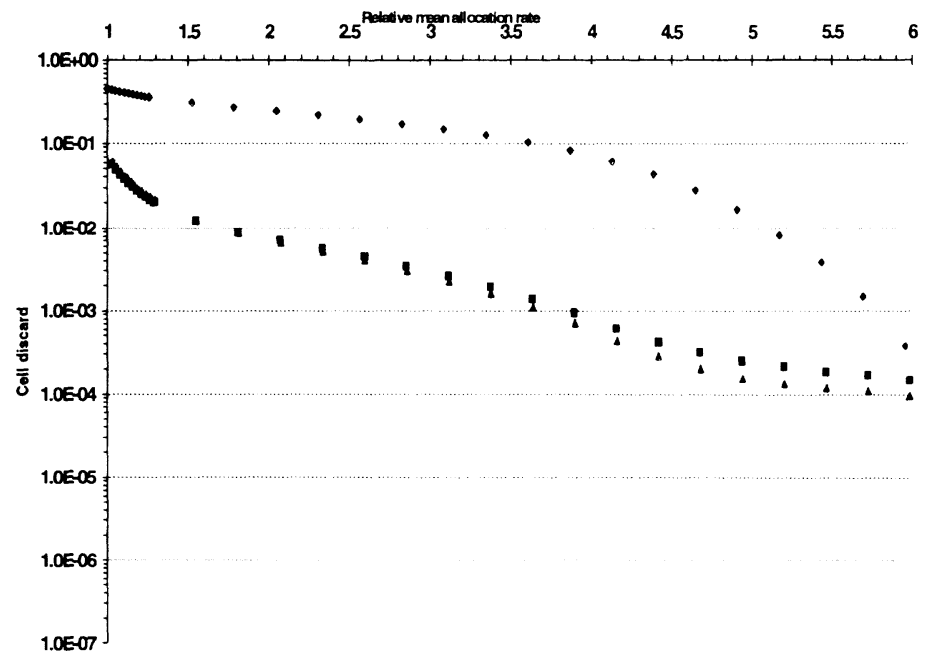

- Classical scheme

- Predictive "Time Series Analysis - EWMA" scheme

\ Predictive "Neural" scheme

Figure 6 Cell discard (Talk show trace) 


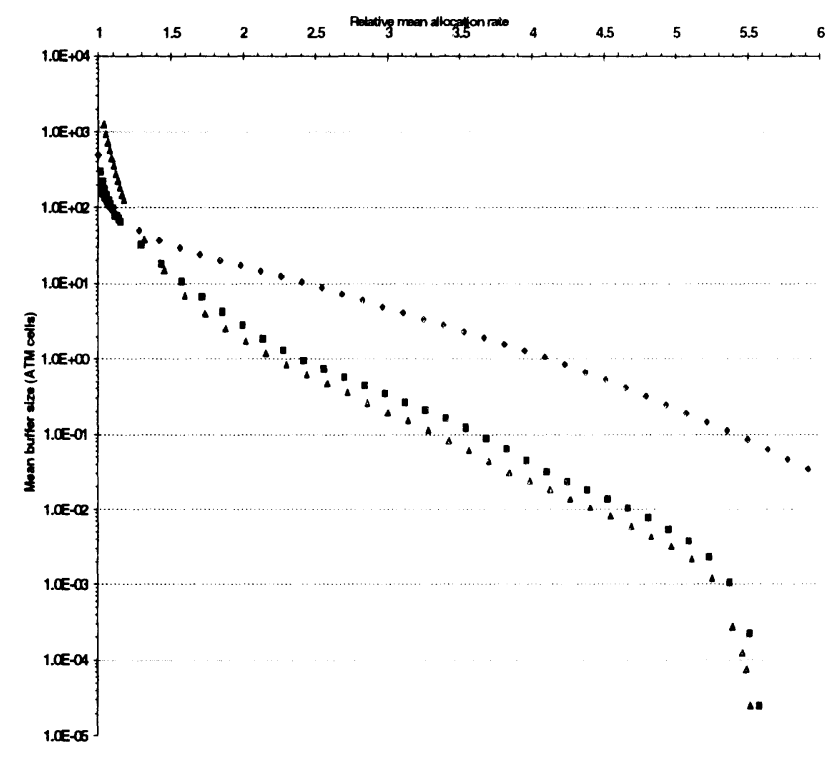

Classical scheme - Predictive "Time Series Analysis - EWMA" scheme

^ Predictive "Neural" scheme

Figure 7 Mean buffer size (Jurassic Park trace)

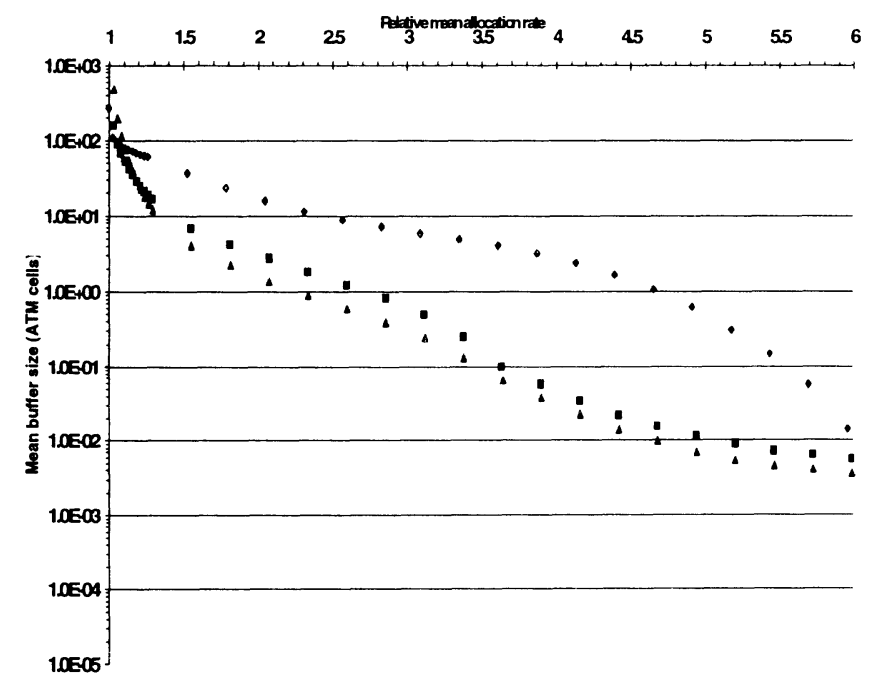

- Classical scheme - Predictive "Time Series Analysis - EWMA" scheme

^ Predictive "Neural" scheme

Figure 8 Mean buffer size (Talk show trace) 